\title{
Periodontal disease increases the host susceptibility to COVID-19 and its severity: a Mendelian randomization study
}

Yi Wang ${ }^{1 \dagger}$, Hui Deng ${ }^{1 \dagger}$, Yihuai Pan' ${ }^{1}$ Lijian Jin², Rongdang Hu' , Yongyong Lư ${ }^{3}$, Wenhai Deng ${ }^{4}$, Weijian Sun ${ }^{5}$, Chengshui Chen ${ }^{6,7}$, Xian Shen $^{5^{*}}$ and Xiu-Feng Huang ${ }^{8,9^{*}}$ (D)

\begin{abstract}
Background: Emerging evidence shows that periodontal disease (PD) may increase the risk of Coronavirus disease 2019 (COVID-19) complications. Here, we undertook a two-sample Mendelian randomization (MR) study, and investigated for the first time the possible causal impact of PD on host susceptibility to COVID-19 and its severity.

Methods: Summary statistics of COVID-19 susceptibility and severity were retrieved from the COVID-19 Host Genetics Initiative and used as outcomes. Single nucleotide polymorphisms associated with PD in Genome-wide association study were included as exposure. Inverse-variance weighted (IVW) method was employed as the main approach to analyze the causal relationships between PD and COVID-19. Three additional methods were adopted, allowing the existence of horizontal pleiotropy, including MR-Egger regression, weighted median and weighted mode methods. Comprehensive sensitivity analyses were also conducted for estimating the robustness of the identified associations.

Results: The MR estimates showed that PD was significantly associated with significantly higher susceptibility to COVID-19 using IVW (OR $=1.024, P=0.017,95 \% \mathrm{Cl} 1.004-1.045)$ and weighted median method $(\mathrm{OR}=1.029, P=0.024$, $95 \% \mathrm{Cl} 1.003-1.055)$. Furthermore, it revealed that PD was significantly linked to COVID-19 severity based on the comparison of hospitalization versus population controls (IVW, $\mathrm{OR}=1.025, P=0.039,95 \% \mathrm{Cl} 1.001-1.049$; weighted median, $\mathrm{OR}=1.030, P=0.027,95 \% \mathrm{Cl} 1.003-1.058)$. No such association was observed in the cohort of highly severe cases confirmed versus those not hospitalized due to COVID-19.
\end{abstract}

Conclusions: We provide evidence on the possible causality of PD accounting for the susceptibility and severity of COVID-19, highlighting the importance of oral/periodontal healthcare for general wellbeing during the pandemic and beyond.

Keywords: COVID-19, Periodontal disease, Risk factor, Mendelian randomization

*Correspondence: 13968888872@163.com; hxfwzmc@163.com

†Yi Wang and Hui Deng contributed equally to this work

${ }^{5}$ Department of Gastrointestinal Surgery, The Second Affiliated Hospital of Wenzhou Medical University, Wenzhou, Zhejiang, China

${ }^{8}$ The Second Affiliated Hospital of Wenzhou Medical University, Wenzhou, Zhejiang, China

Full list of author information is available at the end of the article

\section{Introduction}

Coronavirus disease 2019 (COVID-19), initiated by a novel severe acute respiratory syndrome coronavirus 2 (SARS-CoV-2), has become a global pandemic since March 2020. Despite the strict lockdown measures and implementation of vaccination, the number of COVID19 cases and deaths has reached over 169 and 3 million respectively, according to the WHO report updated on 30 May 2021 [1]. Notably, the susceptibility and severity 
of COVID-19 are highly heterogeneous. Most individuals are presented with asymptomatic infection, while severe cases are developed into acute respiratory distress syndrome, multi-organ failure, and eventually death $[2,3]$. Therefore, identifying potential risk factors for COVID19 is of high priority.

Periodontal disease (PD), comprising gingivitis and periodontitis, is one of the most common immunoinflammatory disorders that result from dysbiosis. Severe periodontitis was estimated to affect $11.2 \%$ of the general population, ranking the sixth most prevalent disease worldwide [4]. PD is characterized by the accumulation of multiple bacterial and subsequent destruction of toothsupporting tissues, leading to tooth loss if left untreated [5]. Accumulating evidence from observational, intervention, and experimental studies has associated PD with a number of comorbidities, such as atherosclerosis, diabetes mellites, respiratory disease, cancer, etc [6, 7]. Moreover, our recent studies suggest that PD is a risk factor for myocardial dysfunction $[8,9]$. The periodontal systemic associations could be briefly explained by the spillover of subgingival pathogens into the bloodstream, as well as a cascade of systemic inflammation and autoimmune damage from the host response [10].

Given the impact of PD on the systemic conditions, it is reasonable to assume that there is a possible linkage between PD and COVID-19. Indeed, several hypotheses have been made based on the previous evidence found in the shared risk factors (e.g., age, obesity, smoking) and bacterial co-infection [11, 12]. Moreover, the mucosal of the oral cavity could be a risky route for SARS-CoV-2 infection since it is highly enriched in the expression of angiotensin-converting enzyme II (ACE2), a major host receptor for SARS-CoV-2 [13]. It is recently demonstrated that patients with painful/bleeding gum are more vulnerable to mortality following COVID-19 infection [14]. Recent case-control studies further identify PD as a contributing factor for COVID-19 infection and complications $[15,16]$. However, these observational studies are prone to confounders, and the causal impacts of PD on host susceptibility to COVID-19 and its severity have not been ascertained.

Mendelian randomization (MR) is a research method that examines the causal relationships between risk factors and disease outcomes by the use of genetic variants as natural experiments [17]. Its principle is comparable to randomized controlled trial (RCT). In RCT, participants are randomly assigned to experimental groups, while in MR, the randomization variable is genetic variants [18]. Although RCT is the gold standard approach to search for causal relationships, a well-designed RCT is expensive, time-consuming, and even impractical. Over the past decade, with Genome-Wide Association
Study (GWAS) summary data being increasingly released for public availability, and developments of statistical approaches, MR analysis is becoming extremely valuable and increasingly applied in many important diseases. MR studies can confirm the associations reported by clinical observational studies, and reveal novel associations [17, 19]. Recently, multiple MR studies have been conducted to infer the causal associations of serval traits on the host susceptibility to COVID-19 and its severity, such as psychiatric disorders [20], glycemic traits [21], and cardiometabolic traits [22]. However, there has been no MR study investigating the potential causal relationships between PD and COVID-19.

Given the aforementioned observational evidence, we hypothesized that periodontal disease may accounts for an increased susceptibility and severity of COVID19. To test our hypothesis, 2-sample MR analyses were performed to investigate the causal inference of genetically predicated periodontal disease on COVID-19 susceptibility and severity. Based on the framework of MR, genetic instruments robustly associated with PD-related Socransky phenotype were retrieved. This phenotype describes a high correlation and positive loading for periodontal pathogens [23]. COVID-19 infection and severe phenotypes, including hospitalized and severe cases from recently conducted GWAS, were included as the outcome of interest [24].

\section{Methods \\ Study design}

This study is a 2-sample MR design, which allows estimating the causal influence of the exposure on the outcome using GWAS summary statistics from two independent studies. The MR design relies on three assumptions (Fig. 1) [25]. First, the genetic instrument should be strongly correlated with PD. Second, the genetic instruments can only be associated with COVID-19 via PD, also known as exclusion restriction assumption. Third, the genetic instruments cannot be associated with any confounders of the exposure-outcome association.

\section{GWAS summary statistics for PD}

The PD-related trait was searched in MR-Base NHGRIEBI GWAS Catalog (https://gwas.mrcieu.ac.uk/) for available GWAS summary statistics. Only those datasets that could be directly downloaded into TwoSampleMR R package for MR analysis (https://github.com/MRCIEU/ TwoSampleMR) were considered. To satisfy the first assumption, the datasets should contain genetic variants that are identified using the genome-wide levels of statistical significance. Finally, only one available dataset with loci that reached genome-wide levels was identified, i.e., PD-related phenotype (Socransky) [23], comprising 


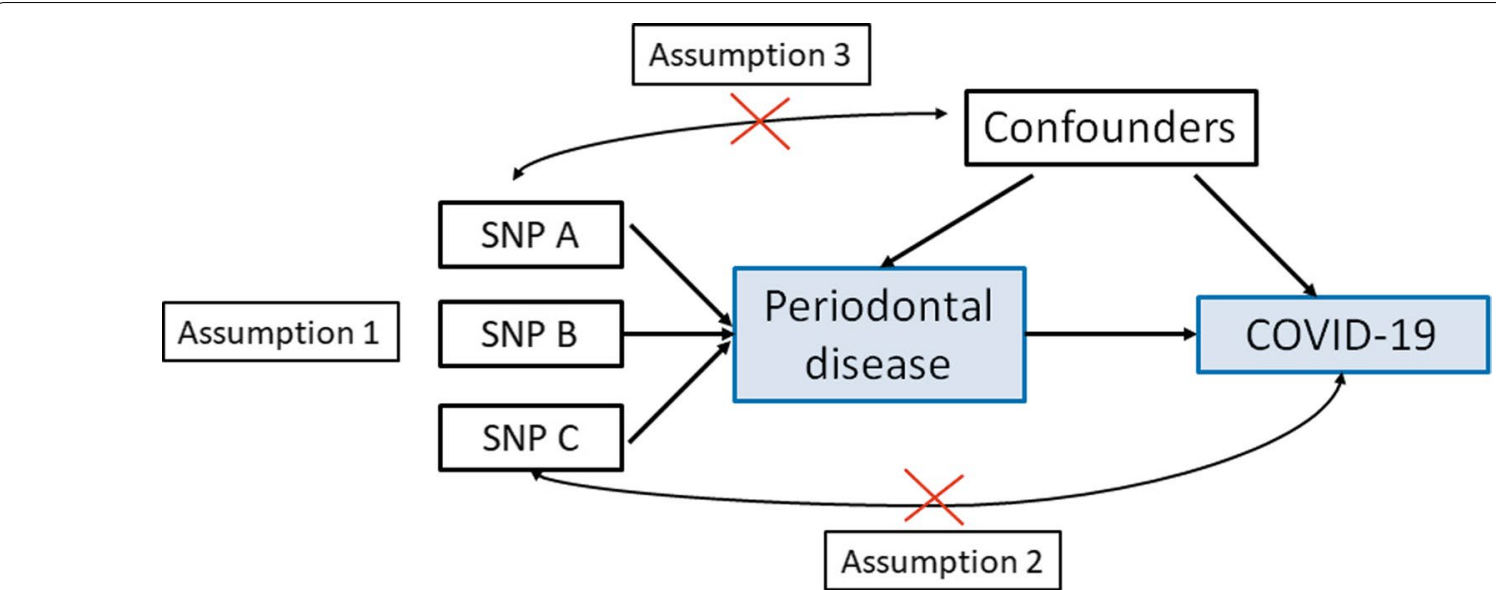

Fig. 1 Principles of MR and the assumptions required to obtain an unbiased causal effect estimate

genotype data on 975 individuals. The detailed description has been summarized in Table 1 .

\section{GWAS summary statistics for COVID-19 disease}

The COVID-19 datasets were from COVID-19 Host Genetics Initiative (https://www.covid19hg.org; Release 4), an international genetics collaboration that aims to discover the genetic determinants of outcomes related to COVID-19 susceptibility and severity [24]. The GWAS results from the comparison of COVID positive versus population controls were used to analyze COVID-19 susceptibility, which comprises 14,134 cases and 1,284,876 controls (Table 1). As for the investigations of COVID19 severity, cohorts based on two different comparisons were used in this study. The first cohort is based on the comparison of hospitalization $(n=6,406)$ versus population controls $(n=902,088)$. The second cohort is from the comparison of very severe respiratory confirmed cases $(n=269)$ versus not hospitalized COVID-19 $(n=688)$ (Table 1).

\section{MR analysis}

Analyses were performed using the $\mathrm{R}$ package TwoSampleMR v0.5.5 (https://github.com/MRCIEU/TwoSa mpleMR) [26]. To guarantee that the association with the exposure was robust (Assumption 1), only SNPs with genome-wide significant variants $\left(P<5 \times 10^{-8}\right)$ were included in the MR analysis. Additionally, variants correlated with the most significant SNPs were removed to ensure only LD-independent genetic variants were remained in the analysis (clumping $r 2$ cut-off $=0.001$ and clumping window $=10,000 \mathrm{~kb}$ ). Subsequently, all included SNPs were harmonized. It indicates that the effect of a SNP on the exposure and the effect of that SNP on the outcome must each correspond to the same allele. Inverse-variance weighted (IVW) method uses a meta-analysis approach to combine Wald estimates for each SNP, effectively treating each SNP as a valid natural experiment. In the present study, the IVW method was adopted as the main analysis for the estimation on the causal relationships of PD with COVID-19. $P$-Value $<0.05$ was considered as the level of significance.

\section{Sensitivity analysis}

Comprehensive sensitivity analyses were performed with the use of TwoSampleMR R package for estimating the robustness of the significant association based on the IVW method $(P$ value $<0.05)$ and potential violations of the MR assumptions (the second and third assumption). Three additional methods were used which allow

Table 1 Description of the GWAS summary statistics

\begin{tabular}{|c|c|c|c|c|c|}
\hline Trait & $\begin{array}{l}\text { GWAS Catalog } \\
\text { accession number }\end{array}$ & Sample size & Number of SNPs & Population & References \\
\hline Periodontal disease (Socransky phenotype) & ebi-a-GCST003484 & 975 & $2,077,804$ & European & {$[23]$} \\
\hline COVID-19 susceptibility (COVID-19 vs. population) & ebi-a-GCST010776 & $1,299,010$ & $11,435,708$ & European & {$[24]$} \\
\hline COVID-19 severity (hospitalized vs. population) & ebi-a-GCST010777 & 908,494 & $12,832,272$ & European & {$[24]$} \\
\hline $\begin{array}{l}\text { COVID-19 severity (very severe respiratory confirmed } \\
\text { vs. not hospitalized) }\end{array}$ & ebi-a-GCST010775 & 957 & $9,201,012$ & European & {$[24]$} \\
\hline
\end{tabular}


the existence of horizontal pleiotropy but has lower statistical power than IVW: (1) MR-Egger regression [27]; (2) Weighted median method [28]; (3) Weighted mode method [26]. In addition to these MR methods, the following sensitivity analyses were also applied in this study: (1) Egger intercept calculation [27]; (2) MR pleiotropy residual sum and outlier (MR-PRESSO) test [29]; (3) Heterogeneity tests [30]; (4) Leave-one-out analysis [26].

\section{Ethical statement}

This study only used publicly available data, and the relevant ethical approval can be found in the corresponding studies referenced in the Methods section.

\section{Results}

\section{MR effect of PD on COVID-19 susceptibility}

Eventually, five SNPs remained for the MR analysis, including rs1156327, rs1633266, rs17184007, rs17718700, and rs3811273. The MR analyses using different methods to estimate the causal inference of PD on COVID-19 susceptibility and severity were presented in Table 2. Interestingly, the MR estimates indicated that PD was significantly associated with COVID-19 susceptibility using the IVW $(\mathrm{OR}=1.024,95 \% \mathrm{CI} 1.004-1.045$, $P=0.017)$ and weighted median method $(\mathrm{OR}=1.029$, 95\% CI 1.003-1.055, $P=0.024$ ) (Table 2). Such causal association was presented in the scatter plot (Fig. 2A). MR Egger and Weighted mode method also showed the same direction of effect $(\mathrm{OR}>1)$, though the $P$ value was not statistically significant. The consistent effects from four different methods suggested that PD could be a risk factor for COVID-19 susceptibility.

Comprehensive sensitivity analyses were then performed to assess the robustness of the causal relationship between PD and COVID-19 susceptibility. An Egger intercept close to zero with $P$ value $>0.05$ suggests there is no directional horizontal pleiotropy. Thus, no evidence of directional horizontal pleiotropy effects was observed using MR Egger intercept test (intercept $=-0.032$, $P=0.76$ ). Second, results from MR-PRESSO test also showed there were no horizontal pleiotropic outliers to distort our result (Global Test $P$-value $=0.304$ ). Additionally, heterogeneity test also suggested that no significant horizontal pleiotropy and heterogeneities $(P$ value of IVW method: $0.26 ; P$ value of MR Egger method: 0.16). Furthermore, there were no outliers among these included 5 SNPs based on leave-one-out analysis. The plot of leave-one-out analysis is shown in Fig. 2B. Taken together, the evidence further supported the causal role of PD on the host susceptibility to COVID-19.

\section{MR effect of PD on COVID-19 severity}

Importantly, the MR estimates demonstrated that PD was also significantly correlated with COVID-19 severity, according to the comparison of hospitalization versus population controls (Table 2, Fig. 3A). Both IVW $(\mathrm{OR}=1.025,95 \% \mathrm{CI} 1.001-1.049, P=0.039)$ and weighted median $(\mathrm{OR}=1.030,95 \%$ CI $1.003-1.058$, $P=0.027)$ methods showed statistical significance. MR Egger and weighted mode methods showed consistent direction of the effect, but $P$ value was not statistically significant. The evidence from comprehensive sensitivity analyses further supported the robustness of the causal association between PD and COVID-19 severity. Egger intercept test indicated that there is no directional horizontal pleiotropy (intercept $=-0.046, P=0.60$ ). Results from MR-PRESSO test showed there were no horizontal pleiotropic outliers to distort our result (Global Test

Table 2 MR results of periodontal disease on risk of COVID-19 susceptibility and severity

\begin{tabular}{|c|c|c|c|c|}
\hline Outcomes & Methods & OR & $(95 \% \mathrm{Cl})$ & $P$ value \\
\hline \multirow{4}{*}{$\begin{array}{l}\text { COVID-19 susceptibility } \\
\text { (COVID-19 vs. population) }\end{array}$} & Inverse variance weighted & 1.024 & $1.004-1.045$ & 0.017 \\
\hline & MR Egger & 1.064 & $0.933-1.214$ & 0.418 \\
\hline & Weighted median & 1.029 & $1.003-1.055$ & 0.024 \\
\hline & Weighted mode & 1.031 & $0.998-1.066$ & 0.135 \\
\hline \multirow{4}{*}{$\begin{array}{l}\text { COVID-19 severity } \\
\text { (Hospitalized vs. population) }\end{array}$} & Inverse variance weighted & 1.025 & $1.001-1.049$ & 0.039 \\
\hline & MR Egger & 1.053 & $0.893-1.240$ & 0.580 \\
\hline & Weighted median & 1.030 & $1.003-1.058$ & 0.027 \\
\hline & Weighted mode & 1.046 & $0.999-1.094$ & 0.139 \\
\hline \multirow{4}{*}{$\begin{array}{l}\text { COVID-19 severity } \\
\text { (Very severe respiratory confirmed vs. not } \\
\text { hospitalized) }\end{array}$} & Inverse variance weighted & 0.964 & $0.797-1.166$ & 0.711 \\
\hline & MR Egger & 1.305 & $0.356-4.784$ & 0.713 \\
\hline & Weighted median & 1.004 & $0.784-1.287$ & 0.967 \\
\hline & Weighted mode & 1.087 & $0.789-1.499$ & 0.652 \\
\hline
\end{tabular}

OR odds ratio, 95\% Cl 95\% confidence interval

Significant associations ( $P$ value $<0.05$ ) are highlighted in bold format 


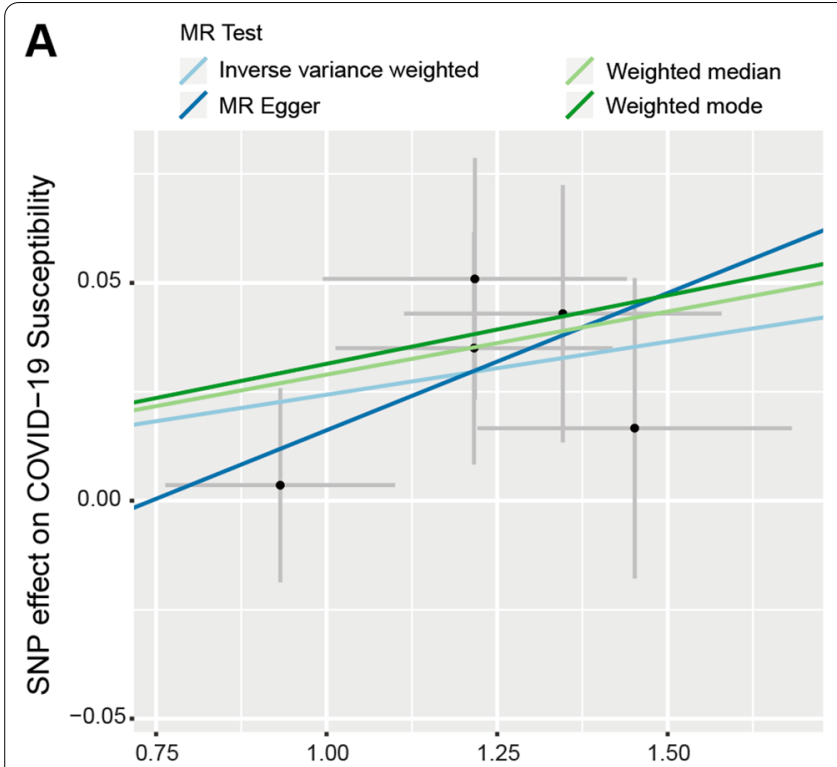

SNP effect on Periodontal disease-related phenotype

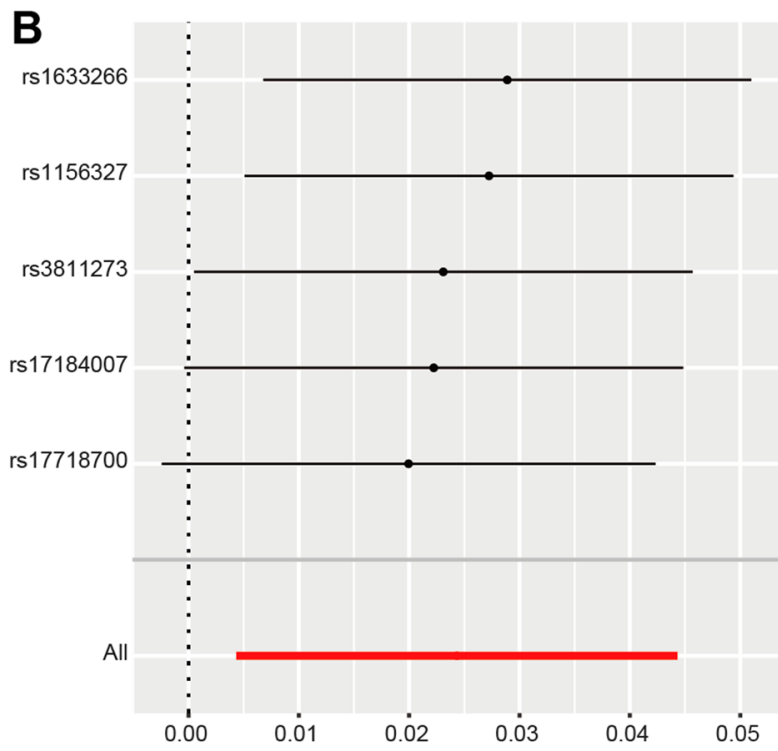

MR leave-one-out sensitivity analysis

Fig. 2 MR analysis and leave-one-out analysis of the causal effect of PD on COVID-19 susceptibility. A Scatter plots for MR analyses of the causal effect of PD on COVID-19 susceptibility. The slope of each line corresponding to the estimated MR effect per method. B Leave-one-out analysis of the causal effect of PD on COVID-19 susceptibility. Each black point represents the IVW MR method applied to estimate the causal effect of PD on COVID-19 susceptibility excluding that particular variant from the analysis. The red point represents the IVW estimate using all SNPS

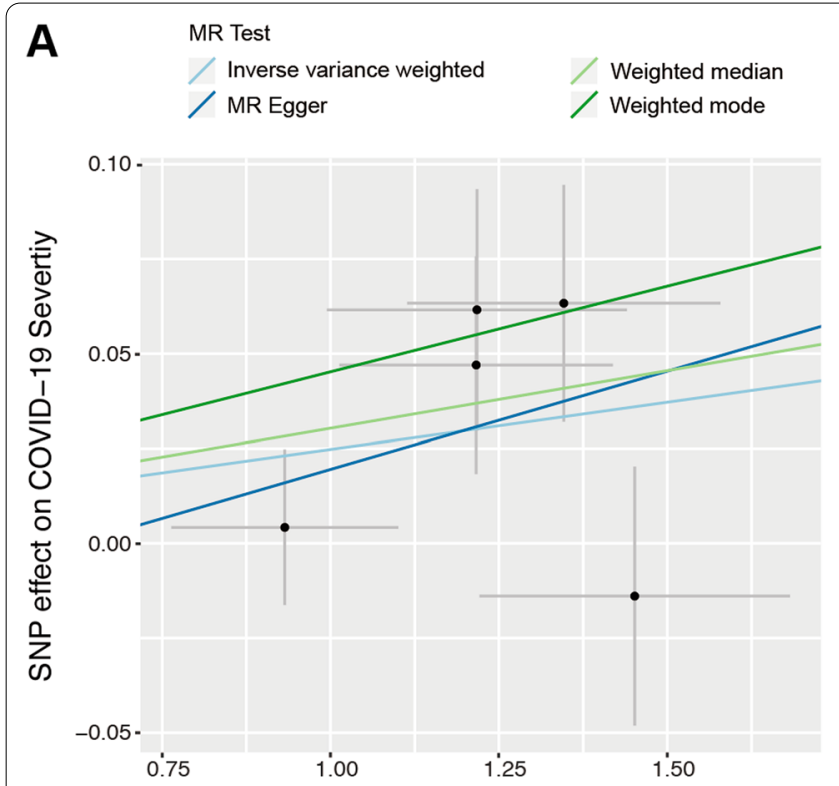

SNP effect on Periodontal disease-related phenotype

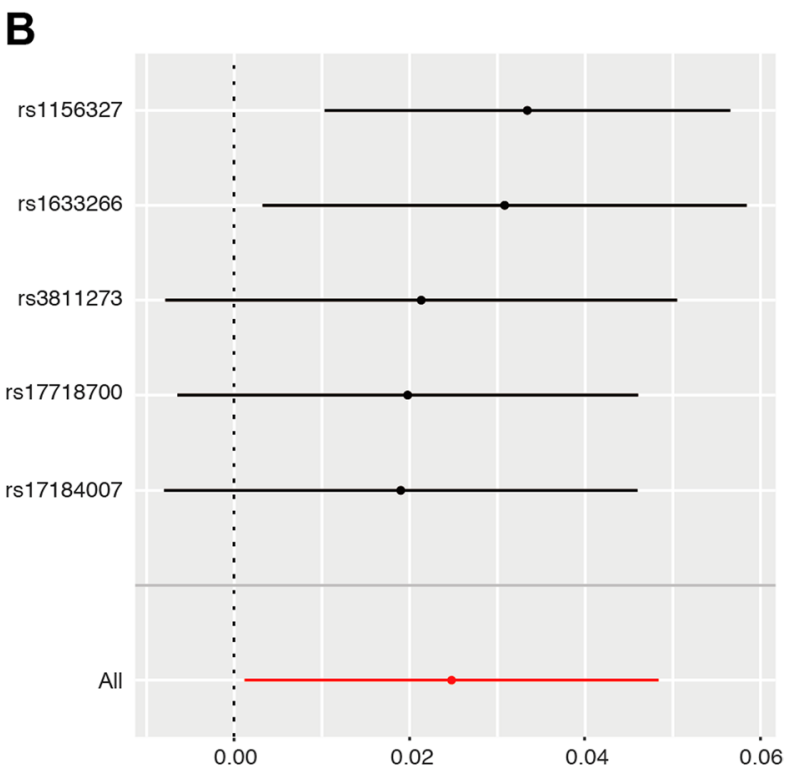

MR leave-one-out sensitivity analysis

Fig. 3 MR analysis and leave-one-out analysis of the causal effect of PD on COVID-19 severity (Hospitalized vs. population). A Scatter plots for MR analyses of the causal effect of PD on COVID-19 severity (Hospitalized vs. population). The slope of each line corresponding to the estimated MR effect per method. B Leave-one-out analysis of the causal effect of PD on COVID-19 severity (Hospitalized vs. population). Each black point represents the IVW MR method applied to estimate the causal effect of PD on COVID-19 susceptibility excluding that particular variant from the analysis. The red point represents the IVW estimate using all SNPS 
$P$-value $=0.783)$. Moreover, heterogeneity test also suggested that no significant horizontal pleiotropy and heterogeneities ( $P$ value of IVW method: $0.77 ; P$ value of MR Egger method: 0.69). In addition, there were no outliers among these included 5 SNPs based on leaveone-out analysis (Fig. 3B). On the contrary, no association between PD and COVID-19 severity was found from the comparison using the cohort of very severe respiratory confirmed cases versus not hospitalized COVID-19 (Table 2).

\section{Discussion}

MR study provides more credible evidence owing to its resistance to confounding with the use of randomly allocated genetic variants at conception. It has been applied to validate whether these risk factors found in the observational findings (e.g., cardiometabolic glycemic and psychiatric trait) have potentially causal effects on COVID-19 [20-22]. To the best of our knowledge, this is the first MR study to gain the inference about the causal role of PD on the susceptibility and severity of COVID-19. Our results suggest that genetically proxied PD is significantly associated with an increased risk of susceptibility and hospitalization of COVID-19. On the contrary, the causal effect of PD on the severe respiratory complication of COVID-19 is not indicated within our current findings.

\section{Literature context}

Our MR findings extend observational evidence suggesting PD is associated with higher susceptibility and severity of COVID-19. In a recent study by Anand et al. [16] they reported a higher susceptibility to COVID-19 in patients with PD (Gingivitis, OR $=17.65$; 95\% CI 5.9552.37; mean clinical attachment loss $\geq 2 \mathrm{~mm}, \mathrm{OR}=8.46$; 95\% CI 3.47-20.63; severe periodontitis, OR $=11.75$; $95 \%$ CI 3.89-35.49). Despite the statistical significance, the wide CIs indicate the low precision of OR, possibly due to the small sample size $(n=150)$. Moreover, given the infeasibility in case/control selection in the pandemic, the case and control groups were complicated by many confounders (e.g., age, hypertension, diabetes, and smoking). Although many of the confounders were adjusted, the residual of confounding may threaten the validity of the estimated OR. On the contrary, no such association was found in another observational study [14]. In this study by Larvin et al., PD was defined as self-reported poor periodontal health from the UK Biobank cohort. However, these questionaries regarding painful gums, bleeding gums and loose teeth are not clinically accurate enough to measure the disease phenotype [31]. These crude measures could be the results of physiological/ pathological factors, such as aging, trauma and previous orthodontic treatment, rather than PD [32].

In a previous study, Marouf et al. compared COVID-19 patients with and without complications and concluded that periodontitis was associated with an increased risk of ICU admission, need for assisted ventilation, and death of COVID-19 patients [15]. In the present study, COVID-19 severity is assessed by hospitalization and severe respiratory complications. The phenotype of hospitalization is based on the comparison between hospitalized COVID-19 individuals and population controls, suggesting that it captures individuals with increased progression into severe symptoms. Therefore, our finding on the association of PD with COVID-19 hospitalization may, at least, in part from the COVID-19 severity. However, the estimated risk for severe respiratory complications in COVID-19 is close to null. Given the small sample size of respiratory confirmed cases $(n=269)$ versus not hospitalized COVID-19 $(\mathrm{n}=688)$, it is possible that MR analysis of COVID-19 respiratory complications is underpowered to detect the small effect of PD, if any.

A number of MR studies have been performed to infer the causal association of genetically predicted periodontitis with systemic commodities including atherosclerosis [33], hypertension [34], cancer [35], and Alzheimer's disease [36]. Unexpectedly, most of these hypotheses were not proven as assessed by MR, possibly due to the limited genome-wide significant variants identified for clinically determined periodontitis [37-40]. In the present study, PD was characterized as periodontal disease relatedSocransky phenotype, suggesting a high correlation and positive loading with pathogens including $P$. gingivalis, P. intermedia, P. nigrescens, T. forsythus, T. denticola, $F$. nucleatum and C. rectus [23]. Such colonization pattern highlights the dysbiotic microbial community, which is closely associated with the most common form of periodontitis [41]. Given the heterogenicity of the clinical manifestation and disease expression among patients with periodontitis, our inclusion of SNPs with PD related Socransky phenotype may better reflect the specific risk of microbial dysbiosis, rather than possible shared risk factors of PD and COVID-19.

\section{Possible mechanisms}

Several hypothetical mechanisms may help explain the association of PD with COVID-19 risk as evidenced by MR. Oral epithelial cells that constitute the buccal and subgingival part of the periodontal pocket are highly enriched in SARS-CoV-2 receptors, namely ACE2 and CD147 [13, 42, 43]. And CD147 is found to be increased in periodontitis patients [44]. Since saliva and gingival crevicular fluids have been shown to be the source for SARS-CoV-2 [45], it is possible that the ulcerated 
epithelium of the periodontal pocket caused by PD, could facilitate the transmission of SARS-CoV-2. In addition, the oral-lung microbiome interactions are wellknown factors contributing to many infectious diseases [46]. Notably, a higher level of oral commensal bacteria has been detected in the bronchoalveolar lavage fluid of COVID-19 patients [47]. It is therefore assumed that periodontopathic bacteria could be frequently aspirated in COVID-19 patients, regulating the mucosal immuneinflammatory response while promoting SARS-CoV-2 infection [12, 48, 49]. Furthermore, PD elevates systemic inflammation by the continuous production of proinflammatory cytokines and chemokines, which potentially aggravate COVID-19 severity $[11,50]$.

\section{Clinical relevance}

The results of this study have some clinical implications for COVID-19 risk management and stratification. To the extent that PD is a modifiable factor, the suspension of dental services to control the spread of the virus could have worsened periodontal health during the pandemic, and it should be viewed with caution. Alternatives such as remote oral hygiene instructions are highly recommended to reduce the bacterial load, maintaining oral/ periodontal health [51]. And the importance of maintaining rigorous oral hygiene should be emphasized, as having PD could be a predisposition to the adverse complications associated with COVID-19. In addition, we recommend poor oral/periodontal health as a risk factor when stratifying individuals who are at higher risk of COVID-19 infection. Accordingly, dentist/periodontist should warn the patients with poor oral hygiene to undertake more rigorous social distancing or shielding. Importantly, vaccines to these high-risk individuals should be prioritized.

\section{Limitations}

Several limitations should be taken into consideration before the interpretation of the results in this MR study. As the sample size of GWAS for PD was small, the main limitation of this study is that it may be underpowered. Although the statistically significant association of PD with COVID-19 susceptibility and severity were identified by different MR methods, the MR estimates were relatively small (OR $=1.024$ for COVID-19 susceptibility, $\mathrm{OR}=1.025$ for COVID-19 hospitalization). It could be explained by the limited SNPs $(\mathrm{n}=5)$ as genetic instruments, which only account for a small proportion in the phenotypic variance of PD. Future GWAS with a larger sample size could assist the discovery of new associations and validation of those findings from this study. Second, the MR findings only reflect the change in COVID-19 risk due to a genetically predisposed (lifetime) status of
PD. In other words, the short-term effect of periodontitis on COVID-19 risk is unknown. Future clinical studies are required to confirm the associations. Additionally, the second cohort $(n=957$; very severe respiratory confirmed cases vs. not hospitalized COVID-19) for the investigations of COVID-19 severity was small. Although no association was observed in this cohort, future study based on larger sample size is necessary.

\section{Conclusions}

Within the limitation of the study, we provide genetic evidence that supports the possible causality of periodontal disease accounting for the host susceptibility to COVID-19 and increased hospitalization rate. Our findings emphasize the great importance of disease prevention and oral/periodontal healthcare for general wellbeing during the pandemic and beyond.

\section{Abbreviations \\ COVID-19: Coronavirus disease 2019; PD: Periodontal disease; MR: Mende- lian randomization; GWAS: Genome-wide association studies; IVW: Inverse variance weighted; MR-PRESSO: MR pleiotropy residual sum and outlier; SARS-CoV-2: Severe acute respiratory syndrome coronavirus 2; SNP: Single nucleotide polymorphism; COVID-19 HGI: COVID-19 Host Genetics Initiative; OR: Odds ratio.}

\section{Acknowledgements}

We are enormously grateful to Offenbacher and his colleagues involved in the study identifying the genetic loci for periodontal disease. We are also extremely thankful to COVID-19 Host Genetics Initiative for making summary statistics publicly accessible for this analysis.

\section{Authors' contributions}

$\mathrm{XFH}, \mathrm{XS}, \mathrm{YW}$ and HD contributed to the study design. XFH and YW contributed to data collection and analysis. All authors contributed to data interpretation. $\mathrm{XFH}$ and $\mathrm{YW}$ wrote the first draft of the manuscript, LJJ revised and refined the manuscript. All authors contributed intellectual material and approved the final draft. All authors had full access to all the data in the study and had final responsibility for the decision to submit for publication. All authors have read and approved the final manuscript.

\section{Funding}

This work was supported by the Natural Science Foundation of Zhejiang Province (LY18H160050, LQ20H140002), Medical and Health Science and Technology Planning Project of Zhejiang Province (2018KY518), National Natural Science Foundation of China (82101029, 31771390, 81972261, 32070151), Wenzhou Science and Technology Bureau (ZY202001-1, ZY2020007, 2020Y0536, Y20190147, Y20190100).

\section{Availability of data and materials}

All data generated or analyzed during this study are included in this published article and its additional material.

\section{Declarations}

Ethics approval and consent to participate

This analysis of publicly available data does not require ethical approval.

Consent for publication

Not applicable.

Competing interests

The authors declare that they have no competing interests. 


\begin{abstract}
Author details
'School \& Hospital of Stomatology, Wenzhou Medical University, Wenzhou, Zhejiang, China. ${ }^{2}$ Faculty of Dentistry, The University of Hong Kong, Hong Kong SAR, China. ${ }^{3}$ Department of Urology, The First Affiliated Hospital of Wenzhou Medical University, Wenzhou, Zhejiang, China. ${ }^{4}$ Key Laboratory of Laboratory Medicine, Ministry of Education, School of Laboratory Medicine and Life Sciences, Wenzhou Medical University, Wenzhou, Zhejiang, China. ${ }^{5}$ Department of Gastrointestinal Surgery, The Second Affiliated Hospital of Wenzhou Medical University, Wenzhou, Zhejiang, China. ${ }^{6}$ Department of Pulmonary and Critical Care Medicine, The First Affiliated Hospital of Wenzhou Medical University, Wenzhou, Zhejiang, China. ${ }^{7}$ Key Laboratory of Interventional Pulmonology of Zhejiang Province, Wenzhou, Zhejiang, China. ${ }^{8}$ The Second Affiliated Hospital of Wenzhou Medical University, Wenzhou, Zhejiang, China ${ }^{9}$ Wenzhou Medical University-Monash BDI Alliance in Clinical and Experimental Biomedicine, Wenzhou Medical University, Wenzhou, Zhejiang, China.
\end{abstract}

\section{Received: 23 August 2021 Accepted: 14 December 2021}

Published online: 24 December 2021

\section{References}

1. https://www.who.int/publications/m/item/weekly-epidemiologicalupdate-on-covid-19---1-june-2021. Accessed 1 July 2021.

2. Zhou F, Yu T, Du R, Fan G, Liu Y, Liu Z, Xiang J, Wang Y, Song B, Gu X, et al. Clinical course and risk factors for mortality of adult inpatients with COVID-19 in Wuhan, China: a retrospective cohort study. Lancet. 2020;395(10229):1054-62

3. Wu Z, McGoogan JM. Characteristics of and important lessons from the coronavirus disease 2019 (COVID-19) outbreak in China: summary of a report of 72314 cases from the Chinese center for disease control and prevention. JAMA. 2020;323(13):1239-42.

4. Kassebaum NJ, Bernabe E, Dahiya M, Bhandari B, Murray CJ, Marcenes W. Global burden of severe periodontitis in 1990-2010: a systematic review and meta-regression. J Dent Res. 2014;93(11):1045-53.

5. Haffajee AD, Socransky SS. Introduction to microbial aspects of periodontal biofilm communities, development and treatment. Periodontol 2000. 2006;42:7-12

6. Falcao A, Bullon P. A review of the influence of periodontal treatment in systemic diseases. Periodontol 2000. 2019;79(1):117-28.

7. Linden GJ, Lyons A, Scannapieco FA. Periodontal systemic associations: review of the evidence. J Clin Periodontol. 2013;40(Suppl 14):S8-19.

8. Wang Y, Zhen Z, Liu HN, Lai I, Pelekos G, Tse HF, Yiu KH, Jin L. Periodontitis links to exacerbation of myocardial dysfunction in subjects with type 2 diabetes. J Periodontal Res. 2019;54(4):339-48.

9. Wang Y, Liu HN, Zhen Z, Pelekos G, Wu MZ, Chen Y, Tonetti M, Tse HF, Yiu KH, Jin L. A randomized controlled trial of the effects of non-surgical periodontal therapy on cardiac function assessed by echocardiography in type 2 diabetic patients. J Clin Periodontol. 2020;47(6):726-36.

10. Hajishengallis G, Chavakis T. Local and systemic mechanisms linking periodontal disease and inflammatory comorbidities. Nat Rev Immunol. 2021. https://doi.org/10.1038/s41577-020-00488-6.

11. Campisi G, Bizzoca ME, Lo Muzio L. COVID-19 and periodontitis: reflecting on a possible association. Head Face Med. 2021;17(1):16.

12. Bao L, Zhang C, Dong J, Zhao L, Li Y, Sun J. Oral microbiome and SARS CoV-2: beware of lung co-infection. Front Microbiol. 1840;2020:11.

13. Xu H, Zhong L, Deng J, Peng J, Dan H, Zeng X, Li T, Chen Q. High expression of ACE2 receptor of 2019-nCoV on the epithelial cells of oral mucosa. Int J Oral Sci. 2020;12(1):8.

14. Larvin H, Wilmott S, Wu J, Kang J. The impact of periodontal disease on hospital admission and mortality during COVID-19 pandemic. Front Med (Lausanne). 2020;7: 604980.

15. Marouf N, Cai W, Said KN, Daas H, Diab H, Chinta VR, Hssain AA, Nicolau B, Sanz M, Tamimi F. Association between periodontitis and severity of COVID-19 infection: a case-control study. J Clin Periodontol. 2021;48(4):483-91.

16. Anand PS, Jadhav P, Kamath KP, Kumar SR, Vijayalaxmi S, Anil S. A casecontrol study on the association between periodontitis and coronavirus disease (COVID-19). J Periodontol. 2021. https://doi.org/10.1002/JPER. 21-0272.
17. Davies NM, Holmes MV, Davey Smith G. Reading Mendelian randomisation studies: a guide, glossary, and checklist for clinicians. BMJ. 2018;362: k601.

18. Zhao SS, Mackie SL, Zheng J. Why clinicians should know about Mendelian randomization. Rheumatology (Oxford). 2021;60(4):1577-9.

19. Prins BP, Abbasi A, Wong A, Vaez A, Nolte I, Franceschini N, Stuart PE, Guterriez Achury J, Mistry V, Bradfield JP, et al. Investigating the causal relationship of C-reactive protein with 32 complex somatic and psychiatric outcomes: a large-scale cross-consortium Mendelian randomization study. PLoS Med. 2016;13(6): e1001976.

20. Luykx JJ, Lin BD. Are psychiatric disorders risk factors for COVID-19 susceptibility and severity? a two-sample, bidirectional, univariable, and multivariable Mendelian randomization study. Transl Psychiatry. 2021:11(1):210

21. Au Yeung SL, Zhao JV, Schooling CM. Evaluation of glycemic traits in susceptibility to COVID-19 risk: a Mendelian randomization study. BMC Med. 2021;19(1):72.

22. Leong A, Cole JB, Brenner LN, Meigs JB, Florez JC, Mercader JM. Cardiometabolic risk factors for COVID-19 susceptibility and severity: a Mendelian randomization analysis. PLoS Med. 2021;18(3): e1003553.

23. Offenbacher S, Divaris K, Barros SP, Moss KL, Marchesan JT, Morelli T, Zhang S, Kim S, Sun L, Beck JD, et al. Genome-wide association study of biologically informed periodontal complex traits offers novel insights into the genetic basis of periodontal disease. Hum Mol Genet. 2016;25(10):2113-29.

24. Initiative $\mathrm{C}-\mathrm{HG}$. The COVID-19 host genetics initiative, a global initiative to elucidate the role of host genetic factors in susceptibility and severity of the SARS-CoV-2 virus pandemic. Eur J Hum Genet. 2020;28(6):715-8.

25. Lawlor DA, Harbord RM, Sterne JA, Timpson N, Davey Smith G. Mendelian randomization: using genes as instruments for making causal inferences in epidemiology. Stat Med. 2008;27(8):1133-63.

26. Hemani G, Zheng J, Elsworth B, Wade KH, Haberland V, Baird D, Laurin C, Burgess S, Bowden J, Langdon R, et al. The MR-Base platform supports systematic causal inference across the human phenome. Elife. 2018. https://doi.org/10.7554/eLife.34408.

27. Bowden J, Davey Smith G, Burgess S. Mendelian randomization with invalid instruments: effect estimation and bias detection through Egger regression. Int J Epidemiol. 2015:44(2):512-25.

28. Bowden J, Davey Smith G, Haycock PC, Burgess S. Consistent estimation in Mendelian randomization with some invalid instruments using a weighted median estimator. Genet Epidemiol. 2016;40(4):304-14.

29. Verbanck M Chen CY, Neale B, Do R. Detection of widespread horizontal pleiotropy in causal relationships inferred from Mendelian randomization between complex traits and diseases. Nat Genet. 2018;50(5):693-8.

30. Bowden J, Del Greco MF, Minelli C, Davey Smith G, Sheehan N, Thompson J. A framework for the investigation of pleiotropy in two-sample summary data Mendelian randomization. Stat Med. 2017;36(11):1783-802.

31. Joshipura KJ, Douglass CW, Garcia RI, Valachovic R, Willett WC. Validity of a self-reported periodontal disease measure. J Public Health Dent. 1996;56(4):205-12.

32. Jordao HW, McKenna G, McMenamin UC, Kunzmann AT, Murray L, Coleman HG. The association between self-reported poor oral health and gastrointestinal cancer risk in the UK Biobank: a large prospective cohort study. United European Gastroenterol J. 2019;7(9):1241-9.

33. Bell S, Gibson JT, Harshfield EL, Markus HS. Is periodontitis a risk factor for ischaemic stroke, coronary artery disease and subclinical atherosclerosis? A Mendelian randomization study. Atherosclerosis. 2020:313:111-7.

34. Czesnikiewicz-Guzik M, Osmenda G, Siedlinski M, Nosalski R, Pelka P, Nowakowski D, Wilk G, Mikolajczyk TP, Schramm-Luc A, Furtak A, et al. Causal association between periodontitis and hypertension: evidence from Mendelian randomization and a randomized controlled trial of nonsurgical periodontal therapy. Eur Heart J. 2019;40(42):3459-70.

35. Corlin L, Ruan M, Tsilidis KK, Bouras E, Yu Y-H, Stolzenberg-Solomon R, Klein AP, Risch HA, Amos Cl, Sakoda LC. Two-sample mendelian randomization analysis of associations between periodontal disease and risk of cancer. JNCI Cancer Spectr. 2021. https://doi.org/10.1093/jncics/pkab037.

36. Sun YQ, Richmond RC, Chen Y, Mai XM. Mixed evidence for the relationship between periodontitis and Alzheimer's disease: a bidirectional Mendelian randomization study. PLoS ONE. 2020;15(1): e0228206.

37. Munz M, Richter GM, Loos BG, Jepsen S, Divaris K, Offenbacher S, Teumer A, Holtfreter B, Kocher T, Bruckmann C, et al. Meta-analysis of 
genome-wide association studies of aggressive and chronic periodontitis identifies two novel risk loci. Eur J Hum Genet. 2019;27(1):102-13.

38. Munz M, Willenborg C, Richter GM, Jockel-Schneider Y, Graetz C, Staufenbiel I, Wellmann J, Berger K, Krone B, Hoffmann P, et al. A genome-wide association study identifies nucleotide variants at SIGLEC5 and DEFA1A3 as risk loci for periodontitis. Hum Mol Genet. 2017;26(13):2577-88.

39. Schaefer AS, Richter GM, Nothnagel M, Manke T, Dommisch H, Jacobs $G$ Arlt A, Rosenstiel P, Noack B, Groessner-Schreiber B, et al. A genome-wide association study identifies GLT6D1 as a susceptibility locus for periodontitis. Hum Mol Genet. 2010;19(3):553-62.

40. Divaris K, Monda KL, North KE, Olshan AF, Reynolds LM, Hsueh WC, Lange EM, Moss K, Barros SP, Weyant RJ, et al. Exploring the genetic basis of chronic periodontitis: a genome-wide association study. Hum Mol Genet. 2013;22(11):2312-24.

41. Socransky SS, Haffajee AD, Cugini MA, Smith C, Kent RL Jr. Microbial complexes in subgingival plaque. J Clin Periodontol. 1998;25(2):134-44.

42. Feldman M, La VD, Lombardo Bedran TB, Palomari Spolidorio DM, Grenier D. Porphyromonas gingivalis-mediated shedding of extracellular matrix metalloproteinase inducer (EMMPRIN) by oral epithelial cells: a potential role in inflammatory periodontal disease. Microbes Infect. 2011;13(14-15):1261-9.

43. Wang K, Chen W, Zhang Z, Deng Y, Lian JQ, Du P, Wei D, Zhang Y, Sun XX, Gong L, et al. CD147-spike protein is a novel route for SARS-CoV-2 infection to host cells. Signal Transduct Target Ther. 2020;5(1):283.

44. Wang J, Yang D, Li C, Shang S, Xiang J. Expression of extracellular matrix metalloproteinase inducer glycosylation and caveolin-1 in healthy and inflamed human gingiva. J Periodontal Res. 2014;49(2):197-204.

45. Gupta S, Mohindra R, Chauhan PK, Singla V, Goyal K, Sahni V, Gaur R, Verma DK, Ghosh A, Soni RK, et al. SARS-CoV-2 detection in gingival crevicular fluid. J Dent Res. 2021;100(2):187-93.

46. Mammen MJ, Scannapieco FA, Sethi S. Oral-lung microbiome interactions in lung diseases. Periodontol 2000. 2020;83(1):234-41.

47. Shen Z, Xiao Y, Kang L, Ma W, Shi L, Zhang L, Zhou Z, Yang J, Zhong J, Yang $D$, et al. Genomic diversity of severe acute respiratory syndromecoronavirus 2 in patients with coronavirus disease 2019. Clin Infect Dis. 2020;71(15):713-20.

48. Takahashi Y, Watanabe N, Kamio N, Yokoe S, Suzuki R, Sato S, linuma T, Imai K. Expression of the SARS-CoV-2 receptor ACE2 and proinflammatory cytokines induced by the periodontopathic bacterium fusobacterium nucleatum in human respiratory epithelial cells. Int J Mol Sci. 2021. https://doi.org/10.3390/ijms22031352.

49. Patel J, Sampson V. The role of oral bacteria in COVID-19. Lancet Microbe. 2020;1(3): e105.

50. Sahni V, Gupta S. COVID-19 and periodontitis: the cytokine connection. Med Hypotheses. 2020;144: 109908.

51. Meng L, Hua F, Bian Z. Coronavirus disease 2019 (COVID-19): emerging and future challenges for dental and oral medicine. J Dent Res. 2020;99(5):481-7.

\section{Publisher's Note}

Springer Nature remains neutral with regard to jurisdictional claims in published maps and institutional affiliations.

Ready to submit your research? Choose BMC and benefit from:

- fast, convenient online submission

- thorough peer review by experienced researchers in your field

- rapid publication on acceptance

- support for research data, including large and complex data types

- gold Open Access which fosters wider collaboration and increased citations

- maximum visibility for your research: over $100 \mathrm{M}$ website views per year

At BMC, research is always in progress.

Learn more biomedcentral.com/submissions 